\title{
Transcriptional regulation of the human, porcine and bovine OCTN2 gene by PPARa via a conserved PPRE located in intron 1
}

\author{
Huidi Luo ${ }^{1 \dagger}$, Yuanqing Zhang ${ }^{1+}$, Huihui Guo ${ }^{1}$, Li Zhang ${ }^{1}, \mathrm{Xi} \mathrm{Li}^{1}$, Robert Ringseis${ }^{2}$, Gaiping Wen², Dequan Hui ${ }^{3}$, \\ Aihua Liang ${ }^{3}$, Klaus Eder ${ }^{2}$ and Dongchang $\mathrm{He}^{1^{*}}$
}

\begin{abstract}
Background: The novel organic cation transporter 2 (OCTN2) is the physiologically most important carnitine transporter in tissues and is responsible for carnitine absorption in the intestine, carnitine reabsorption in the kidney and distribution of carnitine between tissues. Genetic studies clearly demonstrated that the mouse OCTN2 gene is directly regulated by peroxisome proliferator-activated receptor a (PPARa). Despite its well conserved role as an important regulator of lipid catabolism in general, the specific genes under control of PPARa within each lipid metabolic pathway were shown to differ between species and it is currently unknown whether the OCTN2 gene is also a PPARa target gene in pig, cattle, and human. In the present study we examined the hypothesis that the porcine, bovine, and human OCTN2 gene are also PPARa target genes.
\end{abstract}

Results: Using positional cloning and reporter gene assays we identified a functional PPRE, each in the intron 1 of the porcine, bovine, and human OCTN2 gene. Gel shift assay confirmed binding of PPARa to this PPRE in the porcine, bovine, and the human OCTN2 gene.

Conclusions: The results of the present study show that the porcine, bovine, and human OCTN2 gene, like the mouse OCTN2 gene, is directly regulated by PPARa. This suggests that regulation of genes involved in carnitine uptake by PPARa is highly conserved across species.

Keywords: OCTN2, PPARa, Pig, Cattle, Human

\section{Background}

The peroxisome proliferator-activated receptor $\alpha$ (PPAR $\alpha)$ is a ligand-activated transcription factor and serves as an important regulator of lipid metabolism and energy homeostasis [1-4]. Ligands of PPAR $\alpha$ are fatty acids which are released from white adipose tissue during energy deprivation and taken up into tissues during this state or exogenous ligands such as fibrates (e.g., WY-14,643, clofibrate) [5]. Transcriptional regulation of genes by PPAR $\alpha$ is mediated by binding of activated PPAR/retinoid $\mathrm{X}$ receptor (RXR) heterodimers to specific DNA sequences, called peroxisome proliferator response elements (PPRE), thereby stimulating the expression of those genes.

\footnotetext{
* Correspondence: sxnkyhdc@163.com

${ }^{\dagger}$ Equal contributors

${ }^{1}$ Institute of Animal Husbandry and Veterinary Medicine, Shanxi Provincial

Academy of Agricultural Sciences, Taiyuan 030031, P. R. China

Full list of author information is available at the end of the article
}

The PPRE consists of a direct repeat of two copies of a AGGTCA-like sequence separated by a single base, commonly called Direct Repeat 1 (DR1), and has been identified in the promoter, intron and 5'-untranslated region of PPAR target genes [6-10].

Interestingly, several earlier studies repeatedly reported that energy deprivation and treatment with fibrates causes a strong, up to five-fold elevation in the concentration of carnitine in the liver of rats [11-13]. Carnitine plays an important role in lipid and energy metabolism by acting as a shuttling molecule for the translocation of long-chain fatty acids from the cytosol into the mitochondrial matrix, where $\beta$-oxidation occurs. The mechanism underlying this phenomenon remained obscure until it was demonstrated many years later that activation of hepatic PPAR $\alpha$, which is induced by both energy deprivation and fibrate treatment, causes an up-regulation of the novel organic cation 
transporter 2 (OCTN2) in liver cells [14]. OCTN2 is the physiologically most important carnitine transporter facilitating carnitine absorption in the intestine, carnitine reabsorption in the kidney, and carnitine distribution between tissues [15]. In subsequent experiments, convincing evidence for the PPAR $\alpha$-dependency of this effect could be provided by demonstrating that the energy deprivationor fibrate-induced increase in hepatic carnitine concentration and up-regulation of OCTN2 occurs only in wild-type mice but not in PPAR $\alpha$ knockout mice $[16,17]$. Moreover, it could be shown recently that the mouse gene encoding OCTN2 contains a functional PPRE in intron 1 and is therefore directly activated by PPAR $\alpha$ [8].

Besides the abovementioned studies in rats and mice, studies in pigs [18,19], chicken [20] and cattle [21,22] demonstrated that PPAR $\alpha$ activation increases hepatic or cellular carnitine content and up-regulates OCTN2 in the liver or hepatocytes, suggesting that the effect of PPAR $\alpha$ as a regulator of carnitine uptake is well conserved across species [23]. Despite the highly conserved role of PPAR $\alpha$ as an important regulator of lipid metabolism in general, the specific genes under control of PPAR $\alpha$ within each lipid metabolic pathway were shown to differ between species [24], and it is currently unknown whether the OCTN2 gene is also a PPAR $\alpha$ target gene in pig, cattle and human. Comparative sequence analysis revealed that the functional PPRE identified in the mouse OCTN2 intron 1 [8] is completely identical $(100 \%)$ to a putative PPRE present in the porcine, bovine and human OCTN2 intron 1 [23] indicating a high degree of conservation of this sequence and a similar regulation of the OCTN2 gene across these species. However, the functionality of a PPRE cannot be predicted from sequence comparison of closely related species with $100 \%$ certainty but rather has to be thoroughly established by cell culture, reporter gene and gel shift experiments. Based on this we hypothesized that the porcine, bovine, and human OCTN2 gene, like the mouse OCTN2 gene, are directly regulated by PPAR $\alpha$. In this study we therefore performed in silico-analyses and reporter gene and gel shift assays to identify a functional PPRE in the porcine, bovine, and human OCTN2 gene.

\section{Results and discussion}

To study regulation of OCTN2 gene expression by PPAR $\alpha$ in porcine and human cells, we treated porcine PK-15 cells and human HepG2 cells without or with PPAR $\alpha$ agonist WY-14,643. Treatment of both cell lines with WY-14,643 increased mRNA levels of the known PPAR $\alpha$ target gene CPT1 about 5-fold indicating successful activation of PPAR $\alpha$ (Figure 1A). Relative mRNA and protein levels of OCTN2 were also elevated by WY-14,643 about 2-fold both, in porcine PK-15 and human HepG2 cells (Figure 1B and C) indicating that porcine and human OCTN2 are regulated directly by
PPAR $\alpha$ in pigs and humans. Like in porcine and human cells, we have recently shown that mRNA and protein levels of OCTN2 are increased by the PPAR $\alpha$ agonist WY-14,643 about 2.2- and 1.4-fold, respectively, in bovine MDBK cells and co-treatment of MDBK cells with a PPAR $\alpha$ antagonist abolished the effect of WY-14,643 [22] suggesting that bovine OCTN2 is probably also regulated directly by PPAR $\alpha$.

To provide evidence for direct regulation of OCTN2 by PPAR $\alpha$, we next performed in silico-analysis of intron 1 of the porcine, bovine, and human OCTN2 gene using DNA sequences from NCBI Genbank (Accession number XM_003123912 for porcine cDNA, CU372899 for porcine genomic DNA; NM_001046502 for bovine cDNA, AC14966 for bovine genomic DNA; NM_003060 for human cDNA, AC000137 for human genomic DNA) and NUBIScan software (nuclear receptor binding site scanner; [25]). According to this, we observed one putative PPRE, which had $100 \%$ sequence identity with the functional PPRE of the mouse OCTN2 intron 1 (located at 1850 to 1862), each located in the intron 1 of porcine OCTN2 (at position nt 2329 to 2341; Figure 2A), bovine OCTN2 (at position nt 1717 to 1729; Figure 2B), and human OCTN2 (at position nt 2271 to 2283; Figure 2C). The porcine, bovine, and human putative PPRE displayed extreme low p-values $(0.00129,0.00106$, and 0.0007, respectively), indicating that these regions are likely candidates for mediating PPAR $\alpha$-dependent regulation of porcine, bovine, and human OCTN2.

To further examine whether the identified putative PPRE are functional, we generated luciferase reporter gene constructs containing a 228 bp (porcine), a 243 bp (bovine) and a 246 bp (human) fragment, each spanning the putative PPRE of the OCTN2 intron 1 in front of a luciferase gene, by means of positional cloning. Subsequently, we transiently transfected these constructs into HepG2 cells and tested their responsiveness to PPAR $\alpha /$ RXR $\alpha$ co-expression and PPAR $\alpha$ agonist WY-14,643 in dual-luciferase reporter assays. As shown in Figure 3A-C, the luciferase activity of all OCTN2-PPRE constructs was increased dramatically by co-expression of exogenous mouse PPAR $\alpha / R X R \alpha$ and treatment with WY-14,643 (8-fold for the porcine OCTN2-PPRE construct, 43-fold for the bovine OCTN2-PPRE construct, and 6-fold for the human OCTN2-PPRE construct) compared to empty vector. The markedly stronger response of the bovine OCTN2 construct compared to the human and porcine OCTN2 constructs should not be over-interpreted and may be explained by a lower binding of transcriptional repressors to the bovine construct compared to the other constructs. In contrast HepG2 cells transfected with the mutant OCTN2-PPRE constructs, in which the putative PPRE were selectively mutated, resulted in a complete loss of responsiveness to exogenous PPAR $\alpha / R X R \alpha$ and 


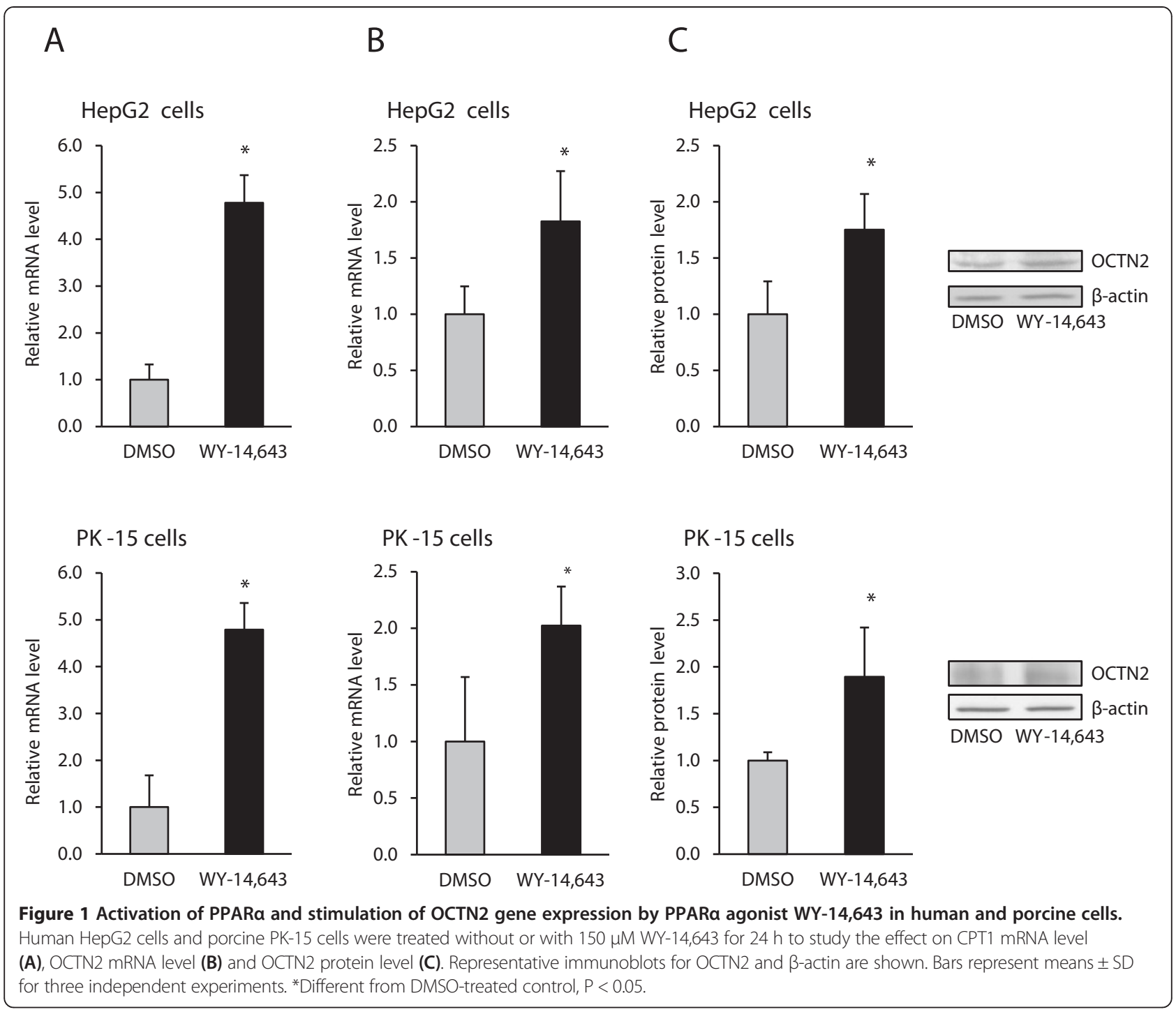

WY-14,643 (Figure 3A-C). These findings indicated that the putative PPRE in the OCTN2 intron 1 is a critical element responsible for PPAR $\alpha$-dependent regulation of the porcine, bovine and human OCTN2 gene.

To finally confirm that this PPRE is functional, we studied in vitro-binding of the PPAR $\alpha / R X R \alpha$ heterodimer to this PPRE using in vitro-translated PPAR $\alpha / \mathrm{RXR} \alpha$ and labeled double-stranded oligonucleotides containing this PPRE sequence in gel shift assays (EMSA). As shown in Figure 4A-C, in the presence of PPAR $\alpha / R X R \alpha$ proteins, a band appeared representing a complex between the labeled oligonucleotide and the PPAR $\alpha / R X R \alpha$ heterodimer (lane 4, Figure 4A-C). On the contrary, no band for the DNA-PPAR $\alpha / R X R \alpha$ complex was observed when an oligonucleotide with a mutation in the putative PPRE was used (lane 5, Figure 4A-C). These observations indicated that the PPAR $\alpha / R X R \alpha$ heterodimer binds specifically to the identified PPRE in the intron 1 of porcine, bovine, and human OCTN2 gene, and this PPRE contributes to PPAR $\alpha$-dependent regulation of the OCTN2 gene in these species.

We have recently postulated that the physiological meaning of PPAR $\alpha$-mediated up-regulation of the OCTN2 gene is to supply cells with sufficient carnitine required for transport of excessive amounts of fatty acids into the mitochondrion, and therefore plays an important role in the adaptive response of cells to PPAR $\alpha$ activation [14]. In cattle and pigs, PPAR $\alpha$ activation physiologically occurs in the liver during early lactation and peak lactation, respectively, because the negative energy balance during these states leads to the release of fatty acids from adipose tissues which are taken up into the liver and bind to and activate PPAR $\alpha$ [26-28]. Thus, the observation from the present study that the porcine and bovine OCTN2 gene is regulated by PPAR $\alpha$ provides a plausible explanation for the recent finding that OCTN2 in the liver is up-regulated 


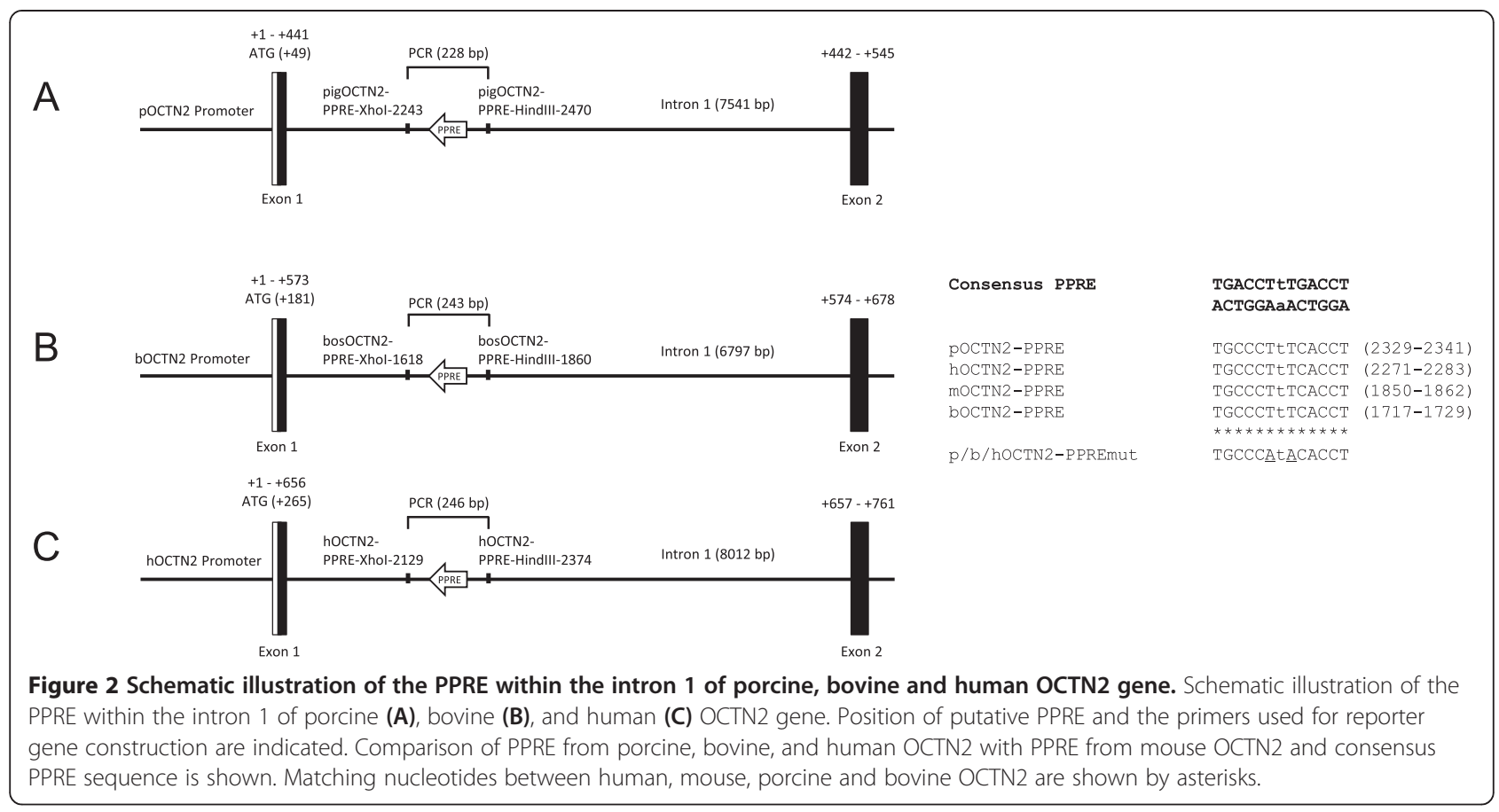

during early lactation in high-producing dairy cows [21] and during peak lactation in sows [28]. Our observation that the human OCTN2 gene is regulated by PPAR $\alpha$ may be not only relevant with regard to carnitine homeostasis but also to tissue distribution and intestinal intestinal absorption of various other compounds, because OCTN2 is polyspecific and able to bind other monovalent cations and various drugs such as verapamil, spironolactone or mildronate [29-31].

\section{Conclusions}

The present results show that the intron 1 of porcine, bovine, and human OCTN2 gene contains a functional PPRE which is critical for PPAR $\alpha$-dependent regulation of the OCTN2 gene. This indicates that the porcine, bovine, and human OCTN2 gene, like the mouse OCTN2 gene [8], are directly regulated by PPAR $\alpha$. Given the fundamental role of OCTN2 for intestinal carnitine absorption and renal carnitine reabsorption and therefore carnitine homeostasis, our findings suggest that PPAR $\alpha$ is a key regulator of carnitine homeostasis in these species. The similar regulation of the OCTN2 gene by PPAR $\alpha$ between mouse, pig, cattle, and human suggests that regulation of genes involved in carnitine uptake by PPAR $\alpha$ is highly conserved across species.

\section{Methods}

\section{Chemicals}

RPMI1640 GlutaMax-1 medium, fetal calf serum and gentamycin were obtained from Invitrogen. WY-14,643 was purchased from Sigma (Beijing, China).

\section{Cell culture}

The human hepatoma cell line HepG2 was obtained from Boster Biological Technology, Ltd. (Wuhan, China). The porcine kidney cell line PK-15 was purchased from Cell Lines Service GmbH (Eppelheim, Germany). HepG2 cells were grown in RPMI1640 GlutaMax-1 medium and PK-15 cells were cultivated in $\mathrm{Hy}$-Clone Minimum Essential Medium/Earle's Balanced Salt Solution (MEM/EBSS) medium. The media were supplemented with $10 \%$ fetal bovine serum and $0.05 \mathrm{mg} / \mathrm{mL}$ gentamicin. All cells were maintained at $37^{\circ} \mathrm{C}$ in a humidified atmosphere of $95 \%$ air and $5 \% \mathrm{CO}_{2}$. Media were changed every 2 days. After reaching a confluence of $70-80 \%$, the cells were either sub-cultivated or used for experiments.

\section{RNA Isolation and qPCR analysis}

For qPCR experiments, cells were seeded in 24-well culture plates at a cell density of $0.7 \times 10^{5}(\mathrm{PK}-15)$ and $1 \times 10^{5}$ (HepG2 cells) per well. After reaching confluence, cells were treated for $24 \mathrm{~h}$ with either $150 \mu \mathrm{M}$ of the PPAR $\alpha$ agonist WY-14,643 or DMSO as vehicle control in lowserum medium $(0.5 \%$ FCS) for HepG2 cells or in medium without serum but with $5 \mathrm{mg} / \mathrm{L}$ bovine insulin for PK-15 cells. Following treatment, total RNA was extracted from cells using Trizol ${ }^{\mathrm{Tm}}$ reagent according to the manufacturer's protocol. cDNA synthesis and qPCR using gene-specific primers were performed as described recently in detail [32]. Gene-specific primers were synthesized by TaKaRa (Dalian, China) and are listed in Table 1. Relative changes in gene expression were calculated using the $2^{-\Delta \Delta C T}$ method [33] using GAPDH as internal control. Normalized 
A

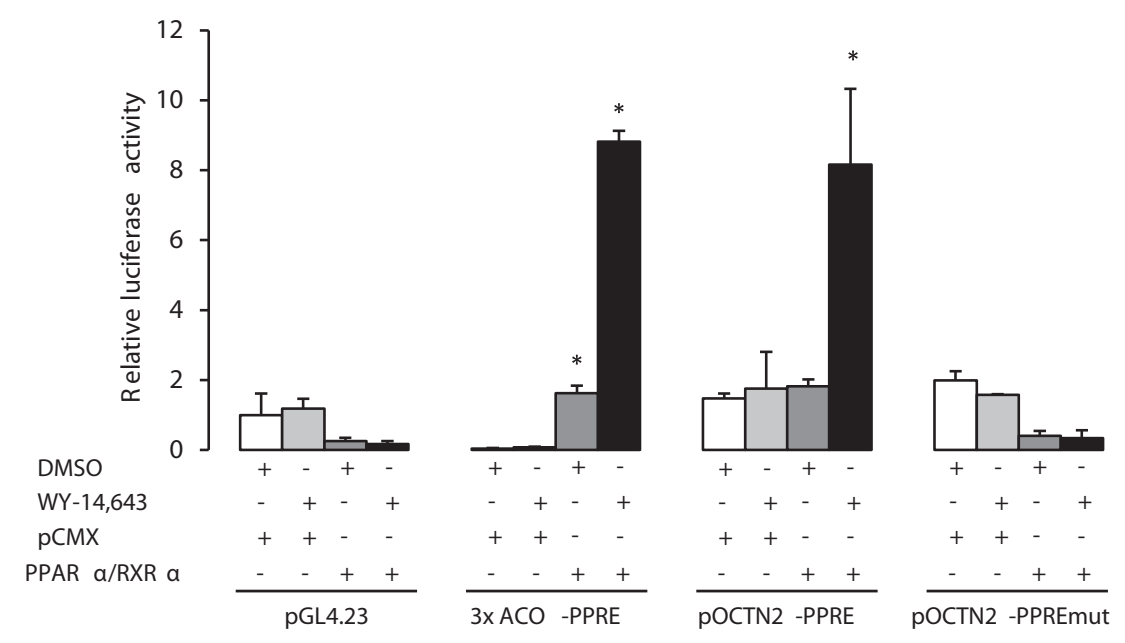

B

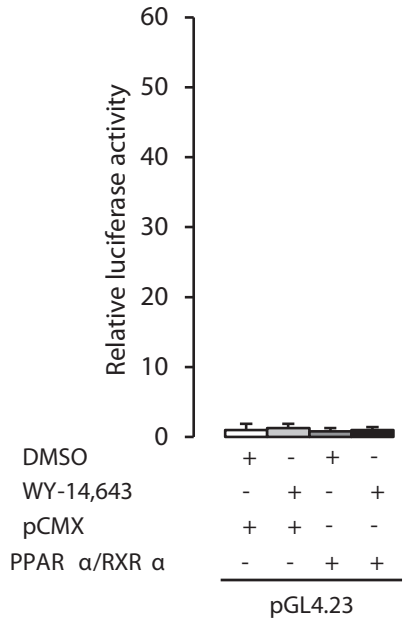

C

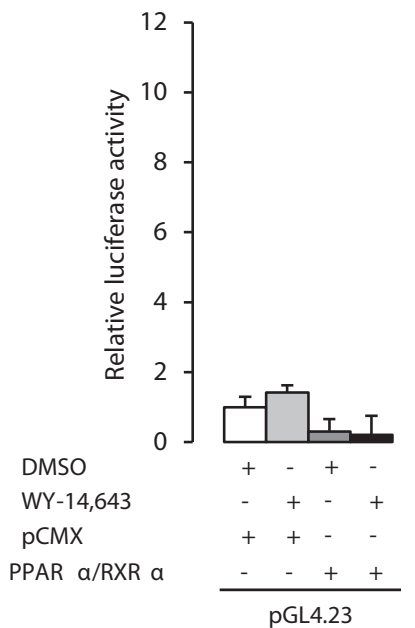

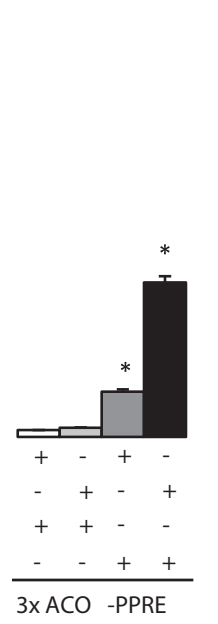
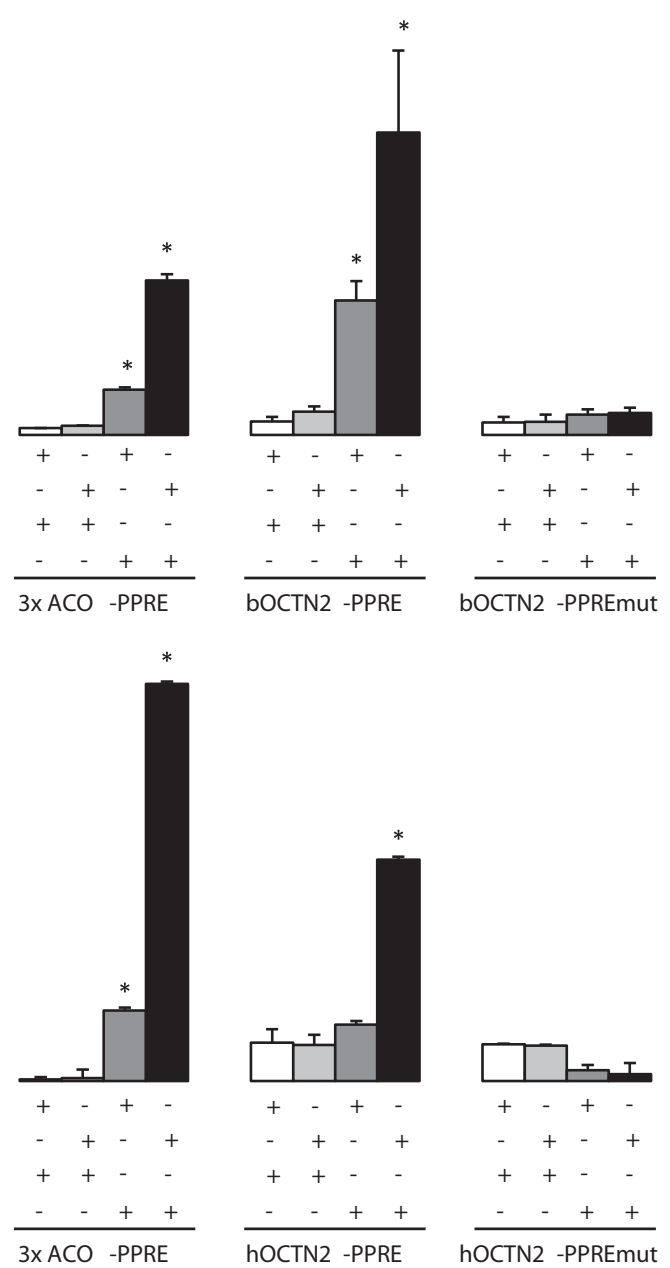

Figure 3 Activation of porcine, bovine and human OCTN2-PPRE reporter gene constructs by PPARa. HepG2 cells were transfected with either (A) wild-type plasmid pGL4.23-pOCTN2-PPRE-Luc or mutant plasmid pGL4.23-pOCTN2-PPREmut-Luc or (B) wild-type plasmid pGL4.23-bOCTN2-PPRE-Luc or mutant plasmid pGL4.23-bOCTN2-PPREmut-Luc or (C) wild-type plasmid pGL4.23-hOCTN2-PPRE-Luc or mutant plasmid pGL4.23-hOCTN2-PPREmut-Luc. Cells were co-transfected with PCMX-mPPARa and pCMX-mRXRa or pCMX (empty vector) expression plasmids, and pGL4.74-RLuc as internal control and normalization plasmid. The plasmids pGL4.23-luc and 3XACO-PPRE were used as negative and positive control plasmids, respectively. $12 \mathrm{~h}$ after transfection cells were cultured in medium in the presence or absence of $50 \mu \mathrm{M} \mathrm{WY}-14,643$ for $24 \mathrm{~h}$ and, subsequently, luciferase activities determined by luminometry. Bars represent means \pm SD for one out of three independent experiments each performed in triplicate. The other experiments revealed similar results. *Different from control (empty vector $\mathrm{pCMX}), \mathrm{P}<0.05$. 


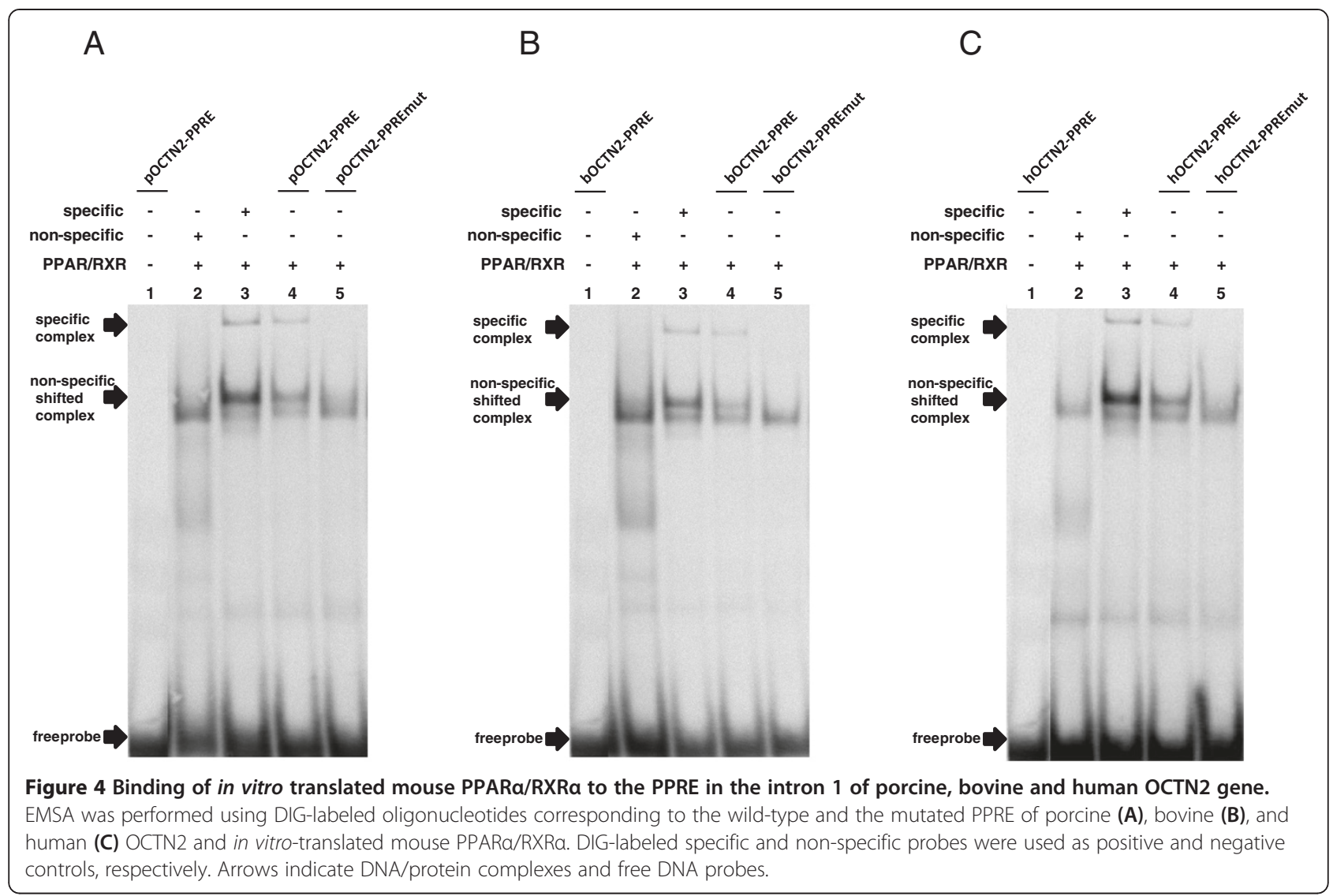

gene expression ratio of WY-14,643 treated cells was presented relative to that of DMSO-treated control cells, which was set to 1.0 .

\section{Western blot analysis}

For western blot experiments, cells were seeded in 6 -well culture plates at a cell density of $2 \times 10^{5}$ (PK-15 cells) and $5 \times 10^{5}$ (HepG2 cells) per well. After reaching confluence, cells were treated as described for qPCR experiments. Following treatment, cells were harvested and lysed with RIPA lysis buffer (50 mM Tris $\mathrm{pH} 7.5$, $150 \mathrm{mM} \mathrm{NaCl}, 1 \mathrm{mM}$ EDTA, 1\% Triton X-100, 0.1\% SDS, $1 \%$ sodium deoxycholate) containing protease inhibitors (Sigma-Aldrich, Steinheim, Germany). Protein concentrations in cell lysates were determined by the
BCA protein assay (Interchim, Montlucon, France). SDS-PAGE was performed with a $4 \%$ stacking gel and a $10 \%$ resolving gel. Afterwards proteins were transferred to a nitrocellulose membrane. The membranes were blocked overnight at $4^{\circ} \mathrm{C}$ in blocking solution (5\% skim milk in Tris buffered saline with Tween-20), and then incubated with mouse monoclonal $\beta$-actin (1:500, Abcom, Cambridge, UK) or mouse polyclonal OCTN2 (1:500, Abcom, Cambridge, UK) primary antibodies for $2 \mathrm{~h}$ at room temperature and overnight at $4^{\circ} \mathrm{C}$, respectively. The membranes were washed with TBS-T $(50 \mathrm{mmol} / \mathrm{L}$ Tris, $150 \mathrm{mmol} / \mathrm{L} \mathrm{NaCl}, \mathrm{pH} \mathrm{7.5,} \mathrm{0.2 \%} \mathrm{Tween-20)} \mathrm{and}$ incubated with a horseradish peroxidase conjugated secondary monoclonal anti-mouse-IgG antibody (1:5000, Jackson Immuno Research, Suffolk, UK) for $1 \mathrm{~h}$ at room

Table 1 Gene-specific primers used for qPCR

\begin{tabular}{llll}
\hline Gene name & Oligonucleotide sequences (forward, reverse) & PCR product size (bp) & Accession number \\
\hline pOCTN2 & TGACCATATCAGTGGGCTA, AGTAGGGAGACAGGATGCT & 384 & XM_003123912 \\
hOCTN2 & GACCATATCAGTGGGCTATTT, CTGCATGAAGAGAAGGACAC & 199 & NM_003060 \\
pGAPDH & AGGGGCTCTCCAGAACATCATCC, TCGCGTGCTCTTGCTGGGGTTGG & 446 & AF017079 \\
hGAPDH & GCCTTCCGTGTCCCCACTGC, CAATGCCAGCCCCAGCGTCA & 211 & NM_002046 \\
pCPT1 & GCATTTGTCCCATCTTTCGT, GCACTGGTCCTTCTGGGATA & 198 & AF288789 \\
hCPT1 & TCACCTCTTCTGCCTTACG, AGTCAAACAGCTCCACTTGC & 132 & NM_001876 \\
\hline
\end{tabular}


temperature. The blots were developed by using the Amersham $^{\text {Tw }}$ ECL Plus Western Blotting Detection System (GE Healthcare, Munich, Germany) and detected by a chemiluminescence imager (Syngene, Cambridge, UK). The signal intensities of specific bands were detected with a Bio-Imaging system (Syngene, Cambridge, UK) and quantified using Syngene GeneTools software. For calculation of protein expression levels, the band intensity of OCTN2 was normalized by that of $\beta$-actin and normalized protein expression level of WY-14,643 treated cells was presented relative to that of DMSOtreated control cells, which was set to 1.0.

\section{Isolation of porcine and bovine genomic DNA}

50-100 mg ear tissues of pig and cattle were obtained from Mashen pig and Jinnan yellow cattle of Shanxi. These experiments were approved by the Shanxi Administration Office of Laboratory Animal. The tissues were washed with $1 \times$ PBS followed by incubation in $500 \mu \mathrm{L}$ lysis buffer containing $100 \mathrm{mM}$ Tris- $\mathrm{HCl}, 5 \mathrm{mM}$ EDTA, 0.2\% SDS, $200 \mathrm{mM} \mathrm{NaCl}$ and $100 \mu \mathrm{g} / \mathrm{mL}$ proteinase $\mathrm{K}$ at $55^{\circ} \mathrm{C}$ overnight. At the next day, the lysate was centrifuged for $5 \mathrm{~min}$ and the supernatant was transferred into a new tube. Subsequently, $500 \mu \mathrm{L}$ of isopropanol was added and centrifuged again for $20 \mathrm{~min}$. Finally, DNA pellets were washed with $70 \%$ ethanol and suspended in TE buffer.

\section{Isolation of human genomic DNA}

For isolation of human genomic DNA, HepG2 cells were seeded in 6-well culture plates at a cell density of $1 \times 10^{6}$ per well. After reaching 70-80\% confluence the medium was removed and the cells were washed with $1 \times$ PBS. Following, cells were collected by scraping and suspended in $500 \mu \mathrm{L}$ lysis buffer containing 5\% SDS, $250 \mathrm{mM}$ Tris and $5 \mathrm{~N} \mathrm{NaOH}$. Subsequently, an equal volume of a 1:1 (v:v) mixture of phenol/chloroform was added and mixed. After a 15 min centrifugation step, the upper aqueous phase was transferred into a new tube containing 100\%

Table 2 Oligonucleotides used for cloning, mutagenesis and EMSA

\begin{tabular}{|c|c|c|c|}
\hline Denomination of oligonucleotides & Oligonucleotide sequences & PCR product size (bp) & Accession number \\
\hline \multicolumn{4}{|l|}{ Oligos used for cloning } \\
\hline pOCTN2-PPRE-HindIII & ATAAAGCTTCAGCCTCTCTGTITCGTCAG & 228 & CU372899 \\
\hline pOCTN2-PPRE-Xhol & ATACTCGAGGAGCTATGTTGCTGCCAGTG & & \\
\hline bOCTN2-PPRE-HindIII & ATAAAGCTTCAGAAGGGTCCTTGAGCTAT & 243 & AC149665 \\
\hline bOCTN2-PPRE-Xhol & ATACTCGAGCCAACAGTGACTGTTCACCA & & \\
\hline hOCTN2-PPRE-HindIII & ATAAAGCTTGCTCTGAACTTCAAGTCAAGC & 246 & AC000137 \\
\hline hOCTN2-PPRE-Xhol & ATACTCGAGCTGAGTGATGGTGGCATTGA & & \\
\hline \multicolumn{4}{|c|}{ Oligos used for mutagenesis and EMSA-mut } \\
\hline pOCTN2-PPREmut-For & AACCTGTAAGTAGGTGTATGGGCACACAACTCGTA & & \\
\hline pOCTN2-PPREmut-Rev & TACGAGTTGTGTGCCCATACACCTACTTACAGGTT & & \\
\hline bOCTN2-PPREmut-For & AACCTGGAAGTAGGTGTATGGGCACAGAGCTCTIT & & \\
\hline bOCTN2-PPREmut-Rev & AAAGAGCTCTGTGCCCATACACCTACTTCCAGGTT & & \\
\hline hOCTN2-PPREmut-For & AACATATAAGTAGGTGtAtGGGCACATAACTCCTT & & \\
\hline hOCTN2-PPREmut-Rev & AAGGAGTTATGTGCCCaTaCACCTACTTATATGTT & & \\
\hline \multicolumn{4}{|l|}{ Oligos used for EMSA } \\
\hline pOCTN2-PPRE-For & AACCTGTAAGTAGGTGAAAGGGCACACAACTCGTA & & \\
\hline pOCTN2-PPRE-Rev & TACGAGTTGTGTGCCCTTTCACCTACTTACAGGTT & & \\
\hline bOCTN2-PPRE-For & AACCTGGAAGTAGGTGAAAGGGCACAGAGCTCTTT & & \\
\hline bOCTN2-PPRE-Rev & AAAGAGCTCTGTGCCCTTTCACCTACTTCCAGGTT & & \\
\hline hOCTN2-PPRE-For & AACATATAAGTAGGTGAAAGGGCACATAACTCCTT & & \\
\hline hOCTN2-PPRE-Rev & AAGGAGTTATGTGCCCTTTCACCTACTTATATGTT & & \\
\hline EMSA-sp.-For & TCTTCCCGAACGTGACCTTTGTCCTGGTCCCCTTT & & \\
\hline EMSA-sp.-Rev & TCAAAGGGGACCAGGACAAAGGTCACGTTCGGGAA & & \\
\hline EMSA-non-sp.-For & TTCCCATCTTGTGAGCTGTCACCCATGGTGGGGTG & & \\
\hline EMSA-non-sp.-Rev & CACCCCACCATGGGTGACAGCTCACAAGATGGGAA & & \\
\hline
\end{tabular}

Abbreviations: $b$ bovine, $h$ human, $p$ porcine. 
ethanol and centrifuged again for 10 min. Finally, DNA pellets were washed with $70 \%$ ethanol and resuspended in TE buffer.

\section{Construction of the reporter genes and site-directed mutagenesis}

A 228 bp DNA fragment spanning 2243 to 2470 in intron 1 of porcine (p) OCTN2 and a 243 bp DNA fragment spanning 1618 to 1860 in intron 1 of bovine (b) OCTN2, each containing a putative PPRE, were amplified by PCR using genomic DNA isolated from Mashen pigs and Jinnan yellow cattle of Shanxi. In addition, a 246 bp DNA fragment spanning 2129 to 2374 in intron 1 of the human (h) OCTN2 gene containing a putative PPRE was amplified by PCR using human genomic DNA isolated from HepG2 cells. Specific primers containing XhoI and HindIII sites for PCR amplification were synthesized by TaKaRa and are listed in Table 2. Each PCR fragment was cloned into the XhoI and HindIII site of the pGL4.23 plasmid (Shanghai Promega Biological Products, Ltd. Shanghai, China) which contains the minimal promoter miniP in front of the luciferase reporter gene luc2. The generated constructs (pGL4.23-pOCTN2-PPRE, pGL4.23-bOCTN2-PPRE, and pGL4.23-hOCTN2-PPRE) were sequenced to confirm absence of any mutation. The mutant luciferase reporter plasmids (pGL4.23-pOCTN2PPREmut, pGL4.23-bOCTN2-PPREmut, and pGL4.23hOCTN2-PPREmut) were generated using Site-Directed Mutagenesis kit according to the manufacturer's protocol (Agilent Technologies Co. Ltd, Beijing, China). Primer sequences used for mutagenesis are shown in Table 2. The mutant constructs were controlled by DNA sequencing.

Transient transfection and dual-luciferase reporter assay Transient transfection was carried out as described previously [6]. Briefly, HepG2 cells were grown in RPMI1640 GlutaMax-1 medium supplemented with $10 \%$ fetal calf serum and $0.05 \mathrm{mg} / \mathrm{mL}$ gentamycin. After reaching 70 $80 \%$ confluency, cells were seeded in 96-well culture plates at a cell density of $4.5 \times 10^{4}$ per well. Then cells were transiently transfected with $50 \mathrm{ng}$ of the generated reporter gene constructs and co-transfected with $50 \mathrm{ng}$ of both, mouse PPAR $\alpha$ expression plasmid (pCMX-mPPAR $\alpha$ ) and mouse RXR $\alpha$ expression plasmid (pCMX-mRXR $\alpha$ ) or 100 ng empty vector (pCMX) using FuGENE 6 transfection reagent (Promega) according to the manufacturer's protocol. Cells were co-transfected with $5 \mathrm{ng}$ pGL4.74 plasmid (Promega) encoding the renilla luciferase reporter gene as internal control reporter vector to normalize for differences in transfection efficiency. A plasmid containing three copies of the functional PPRE from the acyl-CoA oxidase (ACO) promoter in front of a luciferase reporter gene (3xACO-PPRE plasmid) and the empty vector pGL4.23 were transfected as positive and negative controls, respectively. $12 \mathrm{~h}$ after transfection, the cells were treated with either $50 \mu \mathrm{M}$ WY-14,643 to achieve activation of PPAR $\alpha$ or vehicle only $(\mathrm{DMSO}=$ control) for $24 \mathrm{~h}$. Luciferase activities were determined with the DualLuciferase Reporter Assay System from Promega according to the manufacturer's instructions using a Mithras LB940 luminometer (Berthold Technologies, Bad Wildbad, Germany). For control of background luminescence Firefly- and Renilla-luciferase activities were also determined in the lysates of nontransfected control cells and substracted from total luminescence of transfected cells. Data were normalized for transfection efficiency by dividing Firefly-luciferase activity of the generated reporter constructs by that of Renilla-luciferase activity of the cotransfected pGL4.74 Renilla luciferase plasmid. Results represent normalized luciferase activities and are shown relative to cells transfected with the empty vector pGL4.23 and treated with DMSO only which was set to 1 .

\section{Electrophoretic mobility shift assay (EMSA)}

EMSA was performed using DIG Gel Shift Kit, 2nd (Roche) according to the manufacturer's protocol. An oligonucleotide containing the rat ACO-PPRE and a random oligonucleotide were used as specific and nonspecific controls, respectively. Synthesized wild-type and mutated oligonucleotides used for EMSA are listed in Table 1. The mouse PPAR $\alpha$ and RXR $\alpha$ proteins were generated from the expression vectors by in vitro transcription/translation using $\mathrm{TNT}^{\bullet}$ Quick Coupled Transcription/Translation Kit (Promega) according to the manufacturer's protocol. The DNA-protein complexes were detected by chemiluminescence using Anti-Digoxigenin-AP Conjugate and CSPD (Roche) and quantified using a Bio-Imaging system (Syngene, Cambridge, UK) and Syngene GeneTools software.

\section{Statistical analysis}

Quantitative data are expressed as mean \pm standard deviation (SD) based on at least three independent experiments performed in triplicate. Differences were analysed by one-way ANOVA using SAS 9.1.3 (SAS Institute, Inc., 2001). A P-value $<0.05$ was considered to be significant.

\section{Competing interests}

The authors declare that they have no competing interests.

\section{Authors' contributions}

$H L, Y Z, H G, L Z, X L$, and $G W$ carried out the molecular biological studies, participated in the sequence alignment and drafted the manuscript. GW and $\mathrm{DH}$ participated in the sequence alignment. $\mathrm{HL}, \mathrm{YZ}, \mathrm{GW}, \mathrm{DH}$, and $\mathrm{AL}$ participated in the design of the study and performed the statistical analysis. RR, GW, KE, and DoH conceived of the study, and participated in its design and coordination and helped to draft the manuscript. All authors read and approved the final manuscript. 


\section{Acknowledgements}

This study was funded by 100 plans from Shanxi province, national 863 plans projects (2011AA100307-05), and natural science foundation of Shanxi (2012011035-2, 20130311024-1). The funders had no role in study design, data collection and analysis, decision to publish, or preparation of the manuscript.

\section{Author details}

${ }^{1}$ Institute of Animal Husbandry and Veterinary Medicine, Shanxi Provincial Academy of Agricultural Sciences, Taiyuan 030031, P. R. China. ${ }^{2}$ Institute of Animal Nutrition and Nutrition Physiology, Justus-Liebig-University Giessen, 35392 Giessen, Germany. ${ }^{3}$ Key Laboratory of Chemical Biology and Molecular Engineering of Ministry of Education, Institute of Biotechnology, Shanxi University, Taiyuan 030006, P. R. China.

Received: 16 April 2014 Accepted: 6 August 2014

Published online: 08 September 2014

\section{References}

1. Desvergne B, Wahli W: Peroxisome proliferator-activated receptors: nuclear control of metabolism. Endocr Rev 1999, 20:649-688.

2. Chinetti G, Fruchart JC, Staels B: Peroxisome proliferator-activated receptors (PPARs): nuclear receptors at the crossroads between lipid metabolism and inflammation. Inflamm Res 2000, 49:497-505.

3. Guan Y, Zhang Y, Breyer MD: The Role of PPARs in the transcriptional control of cellular processes. Drug News Perspect 2002, 15:147-154.

4. Duval C, Fruchart JC, Staels B: PPARa, fibrates, lipid metabolism and inflammation. Arch Mal Coeur Vaiss 2004, 97:665-672

5. Krey G, Braissant O, L'Horset F, Kalkhoven E, Perroud M, Parker MG, Wahli W: Fatty acids, eicosanoids, and hypolipidemic agents identified as ligands of peroxisome proliferator-activated receptors by coactivator-dependent receptor ligand assay. Mol Endocrinol 1997, 11:779-791.

6. Gutgesell A, Wen G, König B, Koch A, Spielmann J, Stangl Gl, Eder K, Ringseis R: Mouse carnitine-acylcarnitine translocase $(C A C T)$ is transcriptionally regulated by PPARa and PPARס in liver cells. Biochim Biophys Acta 2009, 1790:1206-1216.

7. Mandard S, Müller M, Kersten S: Peroxisome proliferator-activated receptor a target genes. Cell Mol Life Sci 2004, 61:393-416.

8. Wen G, Ringseis R, Eder K: Mouse OCTN2 is directly regulated by peroxisome proliferator-activated receptor a via a PPRE located in the first intron. Biochem Pharmacol 2010, 79:768-776.

9. Wen G, Kühne H, Rauer C, Ringseis R, Eder K: Mouse $\gamma$-butyrobetaine dioxygenase is regulated by peroxisome proliferator-activated receptor a through a PPRE located in the proximal promoter. Biochem Pharmacol 2011, 82:175-183.

10. Wen $G$, Ringseis $R$, Rauer $C$, Eder $K$ : The mouse gene encoding the carnitine biosynthetic enzyme 4-N-trimethylaminobutyraldehyde dehydrogenase is regulated by peroxisome proliferator-activated receptor a. Biochim Biophys Acta 1819, 2012:357-365.

11. Brass EP, Hoppel CL: Carnitine metabolism in the fasting rat. J Biol Chem 1978, 253:2688-2693.

12. McGarry JD, Robles-Valdes C, Foster DW: Role of carnitine in hepatic ketogenesis. Proc Natl Acad Sci U S A 1975, 72:4385-4388.

13. Paul HS, Adibi SA: Paradoxical effects of clofibrate on liver and muscle metabolism in rats. Induction of myotonia and alteration of fatty acid and glucose oxidation. J Clin Invest 1979, 64:405-412.

14. Luci S, Geissler S, König B, Koch A, Stangl Gl, Hirche F, Eder K: PPARa agonists up-regulate organic cation transporters in rat liver cells. Biochem Biophys Res Commun 2006, 350:704-708.

15. Tamai I, Ohashi R, Nezu Jl, Sai Y, Kobayashi D, Oku A, Shimane M, Tsuji A: Molecular and functional characterization of organic cation/carnitine transporter family in mice. J Biol Chem 2000, 275:40064-40072.

16. van Vlies N, Ferdinandusse $S$, Turkenburg M, Wanders RJ, Vaz FM: PPARa-activation results in enhanced carnitine biosynthesis and OCTN2-mediated hepatic carnitine accumulation. Biochim Biophys Acta 2007, 1767:1134-1142.

17. Koch A, König B, Stangl Gl, Eder K: PPARa mediates transcriptional upregulation of novel organic cation transporters- 2 and -3 and enzymes involved in hepatic carnitine synthesis. Exp Biol Med (Maywood) 2008 , 233:356-365.

18. Ringseis R, Luci S, Spielmann J, Kluge H, Fischer M, Geißler S, Wen G, Hirche $\mathrm{F}$, Eder $\mathrm{K}$ : Clofibrate treatment up-regulates novel organic cation transporter (OCTN)-2 in tissues of pigs as a model of non-proliferating species. Eur J Pharmacol 2008, 583:11-17.

19. Ringseis R, Wege N, Wen G, Rauer C, Hirche F, Kluge H, Eder K: Carnitine synthesis and uptake into cells are stimulated by fasting in pigs as a model of nonproliferating species. J Nutr Biochem 2009, 20:840-847.

20. Shibani M, Keller J, Kluge H, König B, Stangl Gl, Ringseis R, Eder K: Effects of activation of peroxisome proliferator-activated receptor- $a$ by clofibrate on carnitine homeostasis in laying hens. African J Agric Res 2012 7:1450-1455.

21. Schlegel G, Keller J, Hirche F, Geißler S, Schwarz FJ, Ringseis R, Stangl GI, Eder $\mathrm{K}$ : Expression of genes involved in hepatic carnitine synthesis and uptake in dairy cows in the transition period and at different stages of lactation. BMC Vet Res 2012, 8:28.

22. Zhou X, Wen G, Ringseis R, Eder K: The pharmacological peroxisome proliferator-activated receptor a agonist WY-14,643 increases expression of novel organic cation transporter 2 and carnitine uptake in bovine kidney cells. J Dairy Sci 2014, 97:345-349.

23. Ringseis $R$, Wen $G$, Eder $K$ : Regulation of Genes Involved in Carnitine Homeostasis by PPARa across Different Species (Rat, Mouse, Pig, Cattle, Chicken, and Human). PPAR Res 2012, 2012:868317.

24. Rakhshandehroo M, Hooiveld G, Müller M, Kersten S: Comparative analysis of gene regulation by the transcription factor PPARa between mouse and human. PLoS One 2009, 4:e6796.

25. Podvinec M, Kaufmann MR, Handschin C, Meyer UA: NUBIScan, an in silico approach for prediction of nuclear receptor response elements. Mol Endocrinol 2002, 16:1269-1279.

26. Loor JJ, Dann HM, Everts RE, Oliveira R, Green CA, Janovick Guretzky NA, Rodriguez-Zas SL, Lewin HA, Drackley JK: Temporal gene expression profiling of liver from periparturient dairy cows reveals complex adaptive mechanisms in hepatic function. Physiol Genomics 2005, 23:217-226

27. Loor JJ: Genomics of metabolic adaptations in the peripartal cow. Animal 2010, 4:1110-1139.

28. Rosenbaum S, Ringseis R, Most E, Hillen S, Becker S, Erhardt G, Reiner G, Eder K: Genes involved in carnitine synthesis and carnitine uptake are up-regulated in the liver of sows during lactation. Acta Vet Scand 2013 55:24

29. Grube M, Meyer zu Schwabedissen HE, Prager D, Haney J, Möritz KU, Meissner K, Rosskopf D, Eckel L, Böhm M, Jedlitschky G, Kroemer HK: Uptake of cardiovascular drugs into the human heart: expression, regulation, and function of the carnitine transporter OCTN2 (SLC22A5). Circulation $2006,113: 1114-1122$

30. Hirano T, Yasuda S, Osaka Y, Kobayashi M, Itagaki S, Iseki K: Mechanism of the inhibitory effect of zwitterionic drugs (levofloxacin and grepafloxacin) on carnitine transporter (OCTN2) in Caco-2 cells. Biochim Biophys Acta 2006, 1758:1743-1750.

31. Kato Y, Sugiura M, Sugiura T, Wakayama T, Kubo Y, Kobayashi D, Sai Y, Tamai I, Iseki S, Tsuji A: Organic cation/carnitine transporter OCTN2 (Slc22a5) is responsible for carnitine transport across apical membranes of small intestinal epithelial cells in mouse. Mol Pharmacol 2006 70:829-837.

32. Ringseis R, Keller J, Lukas I, Spielmann J, Most E, Couturier A, König B, Hirche F, Stangl Gl, Wen G, Eder K: Treatment with pharmacological PPARa agonists stimulates the ubiquitin proteasome pathway and myofibrillar protein breakdown in skeletal muscle of rodents. Biochim Biophys Acta 2013, 1830:2105-2117.

33. Pfaffl MF: A new mathematical model for relative quantification in realtime RT-PCR. Nucleic Acids Res 2001, 29:e45.

doi:10.1186/s12863-014-0090-y

Cite this article as: Luo et al.: Transcriptional regulation of the human, porcine and bovine OCTN2 gene by PPARa via a conserved PPRE located in intron 1. BMC Genetics 2014 15:90 